\title{
Civil Aircraft metal structure superfluous hole repair and stress evaluation method
}

\author{
Weibin $\mathrm{Zhu}^{1^{*}}$, Kai Wang ${ }^{1 *}$, Xiaodong Sun ${ }^{1 *}$, Xue $\mathrm{Yu}^{1 *}$, Jixiang Diao ${ }^{1^{*}}$ \\ ${ }^{1}$ Production Support Engineering Department, Shanghai Aircraft Design and Research Institute, Shanghai, 200436, China
}

\begin{abstract}
Civil aircraft is the most complicated production of the world and during the manufacture various kinds of manufacture nonconformities occur at times. Superfluous hole is one of the most typical nonconformity types. When coping with this kind of problem, engineers often lack experience or standard treatment criteria, which may cause verbose treatment progress, low production efficiency or even technique risk. This article firstly introduces reasons for different types of superfluous holes and figures out treatment procedure and methods and analyses strength evaluation and assessment method.
\end{abstract}

\section{Introduction}

Most of the civil aircraft structure is consisted of aluminium, titanium and steel alloy, using rivet, hi-lock, blind rivet and bolt to connect together.

The number of fasteners on one civil aircraft normally exceeds 10 thousand and fastener holes that differ from design data which are called superfluous holes occur at times. Typical civil aircraft structure consists of skin, panel, stringer, spar, shear clip, angle, corner, bracket, etc. As long as the fastener holes need drilling, superfluous holes are possible to happen.

Superfluous holes have some kinds, such as round holes, singular holes, ellipse holes and "8" shape holes, according to the profile of the hole or the centre distance between the superfluous hole and the right hole.

Reasons of superfluous holes are various, such as workman skill deficiency, work fatigue, inaccurate comprehension of drawings and craft equipment defect.

Superfluous holes can significantly increase stress concentration coefficient and decrease structure life, cause structure failure and even flight danger.

As a result of this, proper repair for superfluous hole is necessary and crucial.

\section{Repair principle}

\subsection{Repair theory}

The goal of superfluous hole repair is to generate compressive stress and lower stress concentration coefficient.

Through transferring compression load that passes the hole and preventing harmful distortion under tension and compression load, the repair will satisfy strength requirements.

\subsection{Repair theory}

Technical treatment for superfluous holes manufacture nonconformity should follow these principles:

- Singular holes should not be plugged before they are rounded [1]. It's crucial to detect crack by no-harm inspection [2]. Besides, burr, metal scrap, greasy dirt and any other foreign object should also be removed;

- Corrosion caused by potential difference should be considered when choosing plugging material. Normally, 2117-T3(AD) rivets or 2024-T3(DD) rivets fit for aluminium, monel(M) rivets fit for steel and titaniumniobium alloy( $\mathrm{T}$ or $\mathrm{U}$ ) rivets fit for titanium. Oppositely, 5056(B) rivets should not be used on 2024 aluminum structure because of potential difference corrosion;

- When the superfluous hole exists on more than one part, the hole should be plugged on each part rather than the several layers, unless the part thickness is less than $0.5 \mathrm{~mm}$;

- When the superfluous hole is close to the right hole, but the net material between two holes is more than 0.05 inch, the superfluous hole can be plugged. If the straight line connecting two hole centers is vertical to the load, structure strengthening should be considered after hole plugging;

- Measures should be adopted for preventing the hole plug from falling off, especially at air pressure borderline. If the hole plugging is between other parts, the repair is safe and stable. But if the hole plugging exists on only one layer of part at air pressure borderline, washer should be applied on the inner side to preventing plug falling off under air pressure;

\footnotetext{
Weibin Zhu: zhuweibin@comac.cc

Kai Wang:wangkai7@comac.cc

Xiaodong Sun: sunxiaodong1@comac.cc

XueYu:yuxue1@comac.cc

Jixiang Diao: diaojixiang@comac.cc
} 
- When the operating space is too limited for riveting, plug with the same material to the base part can be applied to the superfluous hole after freezing in fluid nitrogen or dry ice. The diameter of the freeze plug should be $0.0015-0.0035$ inch $(0.04-0.09 \mathrm{~mm})$ more than that of the superfluous hole. Besides, the freeze plug should be flush with the surface of the part;

- When the superfluous hole is close to the part edge and the net edge distance is less than 0.063 inch, the material between the hole and the edge should be removed because the edge will break during riveting. Rivet plugging is viable only when the net edge distance is more than $0.063 \mathrm{inch}$;

- When plugging holes with countersink, the length of cylinder part of the hole should not be less than 0.5 $\mathrm{mm}$; when the hole plugging is at fatigue sensitive or damage tolerance detail structure, fatigue strength and damage tolerance properties should be re-computed and re-evaluated.

\subsection{Treatment flow chart}

The figure 1 shows the criterion, judgment and treatment options for superfluous holes.

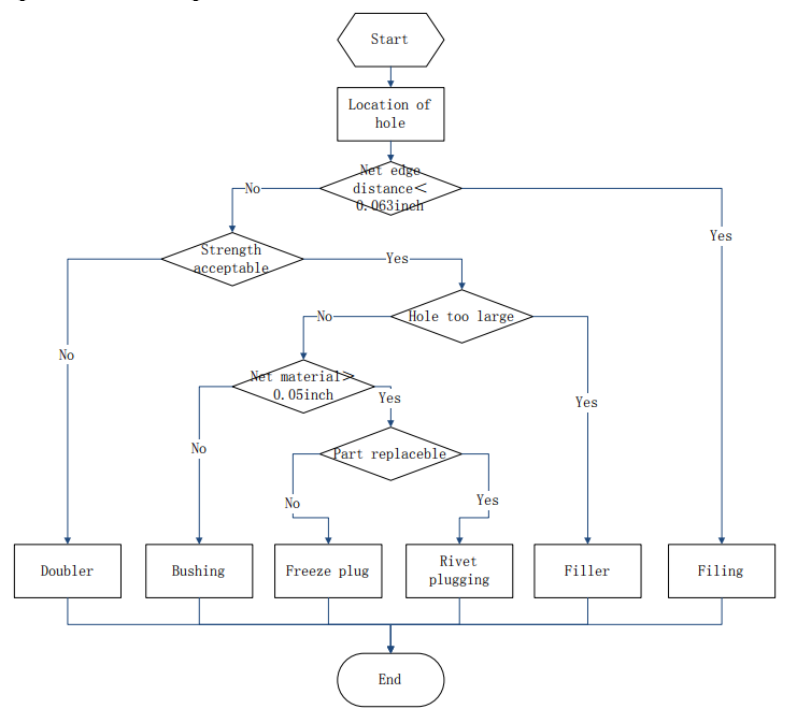

Fig. 1. Flow chart of treatment for superfluous holes.

\section{Treatment method}

\subsection{Rivet plugging}

As a principle, rivet plugging is practicable for holes with diameter less than $8 / 32$ inch, part thickness no less than 0.02 inch and part thickness to hole diameter ratio less than 2. Head rivet plugging and countersink rivet plugging are two basic types of rivet plugging. Countersink rivet plugging consists of one-side and twoside subtypes. Besides, countersink rivet plugging consists of shallow countersink and standard countersink subtypes.

When the superfluous hole exists on only one part and both sides have no other parts to be connected and the part surface does not have to be aerodynamically flush, then head rivet plugging is practicable. The direction of the manufactured head and the riveted rod depends the actual operating environment.

When the superfluous hole exists on more the one part and both outside surface don't have to be flush, then shallow countersink rivet plugging should be added to the inner surface, and the countersink angle is $100 \pm 0.5^{\circ}$ and the countersink depth is $0.2-0.3 \mathrm{~mm}$. The extruded part of the countersink rivet should be milled flush and then cold oxidized.

When the superfluous hole countersink is already made according to the standard dimension, then countersink rivet can be plugged without milling or surface treatment.

When the superfluous hole is at air pressure borderline and exists on at least 2 layers of part, it would be best to replace the inner part. The inner side countersink depth should be increased to $0.5-0.6 \mathrm{~mm}$. Face seal and angle seal should be operated to prevent plug falling off.

If the superfluous hole exists on only one part and the inner side is inaccessible, blind rivet with seal should be used for plugging.

\subsection{Plug plugging}

When the superfluous hole is on more than one layer of part and the parts can't be separated owing to process difficulty or the thickness of the superfluous hole on one part is 2 times more than the diameter, freeze plug should be added.

The freeze plugs should be fixed each by each, with the same length of plug to the thickness of each part. Anti-falling method is necessary and the outside part should be replaced by a new one or additional cover should be added.

Just like the rivet, the plug consists of cylinder plug and cylinder-countersink plug subtypes.

The diameter of the plug should be 0.00150.0035 inch more than the superfluous hole. Each plug should be flush with each part and surface treatment eliminating the cylinder surface is the same as the original part.

\subsection{Filler repair}

When the superfluous hole is on only one layer of part and the diameter is more than $8 / 32$ inch, filler repair should be added to cover the hole [3].

The material, surface treatment and thickness of the filler should be the same as the original part. The filler should be connected using rivets or hi-lock fasteners and fasteners edge distance is at least 2 times of the connecting fastener hole diameter.

The center area may be filled with seal considering the environment, waste fluid and corrosion.

\subsection{Bushing repair}

When the net material between the superfluous hole and the right hole is less than 0.05 inch, the superfluous hole 
and the right hole should be rounded together. At this time, the diameter is much more than the original hole and rivet plugging is not practicable and should be replaced by bushing repair.

Firstly, a plug with the diameter 0.0015-0.0035 inch more than the rounded hole should be manufactured and fixed after freezing [4]. Then, the fastener hole should be drilled in the center of the plug, while the bushing wall thickness should not be less than 0.03 inch.

The bushing repair consists of cylinder bushing, cylinder-countersink bushing and shoulder bushing subtypes.

If the fastener is too small to cover the bushing, washer or shoulder bushing should be added to prevent the bushing from falling off.

\subsection{Doubler repair}

When the diameter of the superfluous hole is more than $8 / 32$ inch, or the hole is evaluated to be incapable of satisfying strength requirements, doubler should be added upon the superfluous hole.

The thickness of the doubler should be no less than that of the reinforced part and the direction of the doubler should be along with the load.

At least 3 fasteners on each side of the superfluous hole is necessary.

\subsection{Filing repair}

When the superfluous hole is close to the edge of the part and net edge distance is less than 0.063 inch, the material close to the edge should be filed. The filing outline should be smooth and filing scope should be 20 times of the filing depth.

Besides, the filing surface roughness and surface treatment should accord with the original part.

\section{Strength evaluation}

\subsection{Static strength evaluation}

The plug superseding the original removed material transfers the load through the part by cooperative distortion, thus causing no influence to the original structure. Besides, the plug does not transfer load between 2 layers of part or cause hole wall extrusion strength problem.

So, plugging is not relevant to static strength problem.

\subsection{Fatigue strength evaluation}

\subsubsection{Summary of fatigue strength evaluation}

For fatigue sensitive detail location, fatigue strength for plugging and other detail during 2 times diameter scope area of plugging should be evaluated meanwhile after plugging repair.

\subsubsection{Plugging fatigue strength computation}

CFQ is the coefficient describing the fatigue property of the specific structure connection, related to material, heat treatment, surface treatment, hole condition, fastener type, etc [5].

CFQ computation for plugging is according to the following equation.

$$
C F Q_{\text {plug }}=C F Q_{\text {hole }} \times \frac{K}{K_{t g}}
$$

in which:

$\mathrm{CFQ}_{\text {plug }}-\mathrm{CFQ}$ after plugging, $\mathrm{MPa}$;

$\mathrm{CFQ}_{\text {hole }}-\mathrm{CFQ}$ of void hole, $\mathrm{MPa}$;

$\mathrm{K}$ - stress concentration coefficient of void hole; $\mathrm{K}=3.1$ for structure bearing tensile load, $\mathrm{K}=4.2$ for structure bearing shear load;

$\mathrm{K}_{\mathrm{tg}}$ - stress concentration coefficient after plugging.

If the side edge distance, end edge distance or hole spacing of the plugging doesn't satisfy relevant requirements, CFQ should be modified according to the following equation.

$$
C F Q_{\text {plug mod }}=C F Q_{\text {plug }} \times M \text { in }\left\{\begin{array}{l}
\text { side edge distance coefficient } \\
\text { end edge distance coefficient } \\
\text { hole spacing coefficient }
\end{array}\right\}
$$

in which:

$\mathrm{CFQ}_{\text {plug mod }}$-modified CFQ, MPa;

$\mathrm{CFQ}_{\text {plug }}-\mathrm{CFQ}$ after plugging, $\mathrm{MPa}$.

Side edge distance coefficient, end edge distance coefficient and hole spacing coefficient are according to figure 2,3 and 4 .
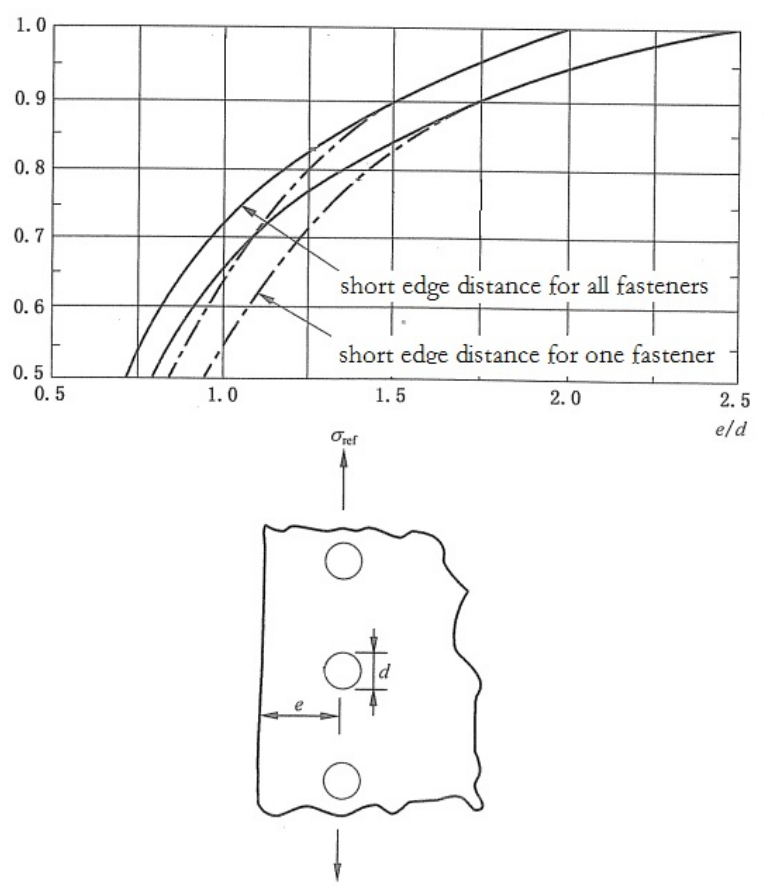

Fig. 2. Side edge distance coefficient 

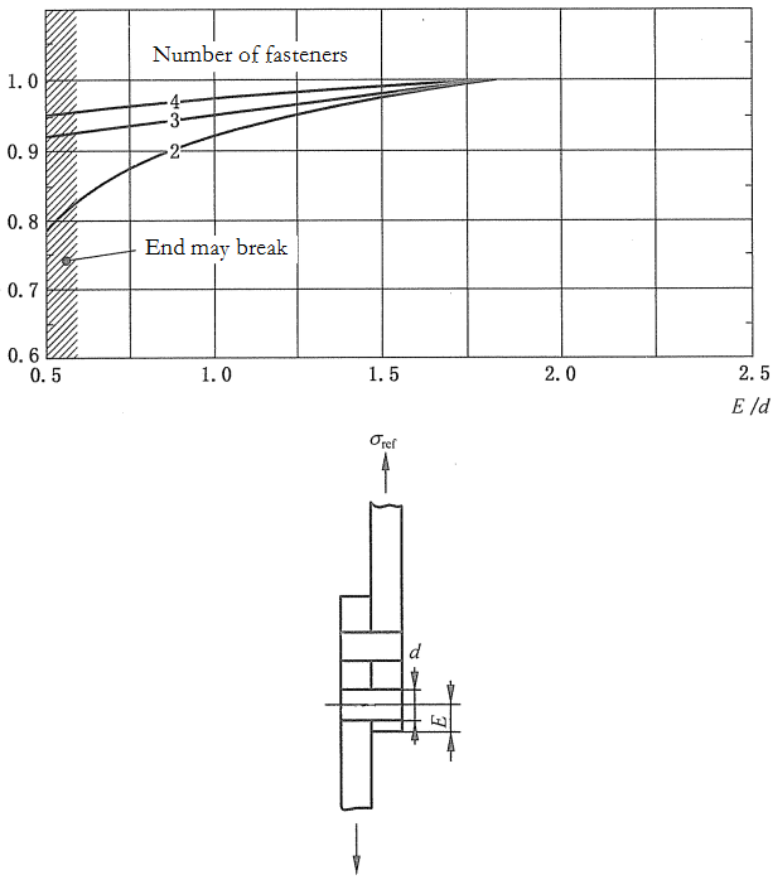

Fig. 3. End edge distance coefficient
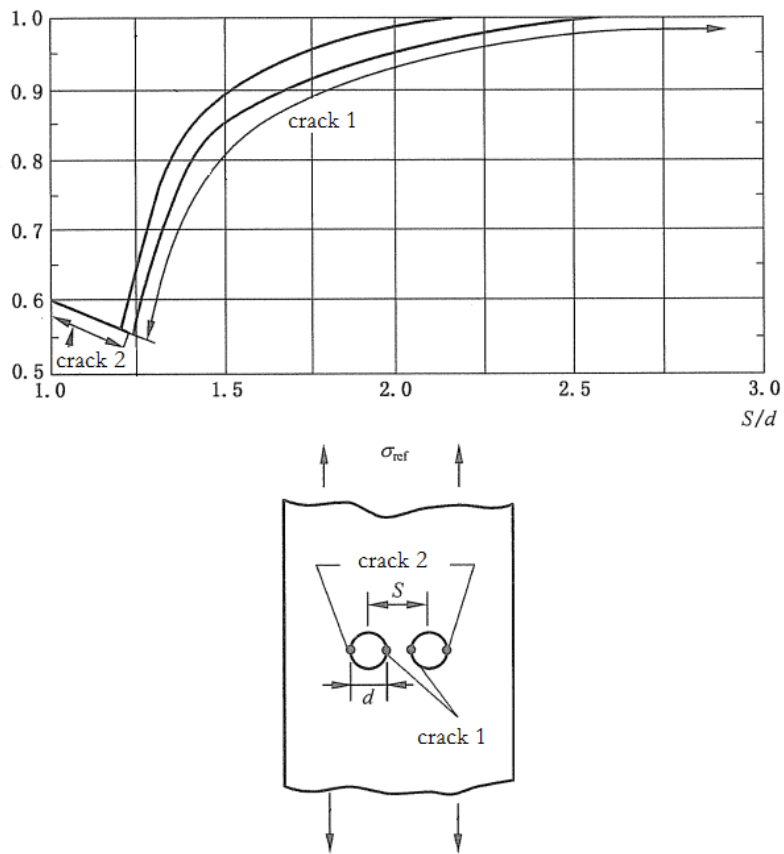

Fig. 4. Hole spacing coefficient

\subsubsection{Fatigue strength computation for 2 time hole diameter of area around plugging}

When the plugging is less than 2 time hole diameter from other fatigue detail, the influence to the original stress concentration coefficient must be considered. The modified CFQ is according to the following equation.

$$
C F Q_{\text {plug mod }}=C F Q_{\text {plug }} \times \frac{K_{\text {tg mod }}}{K_{\text {tg }}}
$$

in which:

$\mathrm{CFQ}_{\text {plug mod }}-$ modified CFQ, MPa;
$\mathrm{CFQ}_{\text {plug }}-\mathrm{CFQ}$ after plugging, $\mathrm{MPa}$;

$\mathrm{K}_{\mathrm{tg} \text { mod }}$-modified stress concentration coefficient;

$\mathrm{Ktg}$ - original stress concentration coefficient.

\subsection{Damage tolerance assessment}

For damage tolerance assessment of plugging, only local stress concentration influence should be modified based on the fact that plugging does not influence crack expand of circumjacent detail.

Considering stress concentration influence, modify the stress magnification factor, as shown in figure 5, as computed in the following equation:

$$
S_{0 \text { mod }}=S_{0} \times \frac{K}{3}
$$

in which:

$\mathrm{S}_{\text {mod }}$-modified stress magnification factor;

$\mathrm{S}_{0}$ - stress magnification factor;

$\mathrm{K}$-stress concentration coefficient for void hole, $\mathrm{K}=3.1$ for structure bearing tensile load.

\section{Conclusion}

This article firstly introduces reasons for superfluous holes and three typical types of superluous holes. Then the article analyses harm of superfluous holes for the aircraft. Then the article figures out treatment principles and six types of treatment methods. In the end, this article analyses strength evaluation and assessment method, mainly including static strength, fatigue strength and damage tolerance.This article can guide manufacture nonconformity treatment during aircraft production.

\section{References}

1. Weina Li, Nonconformity hole repair of civil aircraft, Technology vision, 7:256 (2017)

2. Soufiane Bouarfa, Towards Automated Aircraft Maintenance Inspection. A use case of detecting aircraft dents using Mask R-CNN, AIAA Scitech 2020 Forum (2020)

3. Feng Yan, Development of experimental platform for repairing skin crack patch in aircraft under the teaching environment of skill training, Experimental technology and management, $36: 198-201(2019)$

4. Jiansheng Zou, Influence of repairing technique by bushing on fatigue performance of metal structure for civil aircraft, Civil aircraft design \& research,128:67-70(2018)

5. Xinkang Zhai, Application and discussion of the methods of the rivet removal in aircraft structure repair, Machinery design \& manufacture, 2: 263(2017) 